\title{
Processing feature density in preattentive perception
}

\author{
STEVEN TAYLOR and DAVID BADCOCK \\ University of Melbourne, Parkville, Melbourne, Australia
}

\begin{abstract}
According to the texton theory (Julesz, 1984a, 1984b), preattentive perception is mediated by the detection of density differences of features known as textons. The present study examined this assumption in the case of textons defined by ends of lines (terminators). This was done using a reaction time task in which subjects searched for a target of high terminator density embedded in arrays of lower terminator density. Results indicated that if density differences are utilized in preattentive target detection, then preattentive perception occurs only for particular highly practiced subjects, and then only for large density differences. When terminators are preattentively detected, observers appear to more typically detect only their presence, without computing density differences per se. Implications for other classes of textons were also considered.
\end{abstract}

Preattentive perception is said to occur when the detection of particular feature states occurs effortlessly or instantaneously, without the need for scanning eye movements (Julesz, 1984a, 1984b). Phenomenologically, when these features are present in a visual image, they "pop out," thus drawing focused attention (Hurlbert \& Poggio, 1985). The texton theory (Julesz, 1980, 1981, 1984a, 1984b) postulates that preattentive perception is mediated by the detection of features known as textons. Textons include elongated blobs, the endpoints of very elongated blobs (terminators), and the regions of intersection of very elongated crossed blobs (crossings). Blobs can be further specified in terms of a number of variables, such as length, width, orientation, color, and contrast (Julesz, 1981; Taylor, 1986; Taylor \& Stanley, 1986).

The texton theory entails what will be called the densityprocessing model. According to this model, target detection or texture discrimination is made on the basis of preattentive mechanisms that count the number of textons and calculate density differences (Julesz, 1981, 1984b; Julesz \& Bergen, 1983). The detection of density differences is said to occur over domains as small as a single texture element or micropattern, and to occur regardless of the number of textons in the array (Julesz \& Bergen, 1983). Although the density-processing model has yet to be adequately specified, Julesz (1981) proposed that density differences could be detected by means of comparisons made between texton "counting pools." For a given class of texton, specified according to the above-mentioned at-

Portions of this article were presented at the 14th Annual Experimental Psychology Conference, May 1987, University of New England, Australia. The authors thank Rod Dickinson for his programming assistance and Elizabeth Hinton for the many hours she contributed as a subject. Thanks are also given to Charles W. Eriksen and two anonymous reviewers for their useful comments on previous drafts of this paper. Requests for reprints should be addressed to Steven Taylor, Department of Psychology, University of British Columbia, 2136 West Mall, Vancouver, British Columbia V6T 1Y7, Canada. tributes (e.g., size, contrast), these pools count textons from a given area of the image. This area may be termed the sampling area of a given texton counting pool. For a preattentive system to have sufficient power to function as an "early warning system" for attentive perception (Julesz, 1981, 1984a, 1984b; Treisman \& Souther, 1985), there are probably a number of different sampling areas for a given type of texton (or some functionally analogous mechanism), so that the detection of density differences is not unduly constrained according to parameters such as the size and separation of texture elements.

The texture pairs that have been used to support the density-processing model consist of textures whose respective micropatterns differ in texton number. For example, Julesz (1982, Figure 9) used a texture composed of E-shaped micropatterns and a texture composed of S-shaped micropatterns. Each E-shaped micropattern contained three terminators, whereas each S-shaped micropattern had only two. All micropatterns were the same size, so differences in the number of textons in each type of micropattern corresponded to differences in texton density. Since the two textures were found to be preattentively discriminable, Julesz (1982) concluded that this was the result of differences in terminator density of the respective micropatterns.

In that example, however, the basis of discriminability is open to dispute. Rather than being derived from differences in the relative density, of textons, preattentive texture segregation may have arisen from a more primitive process, which simply signals the presence of feature(s) unique to one of the textures, such as the relatively long line segment forming the spine of each $E$ or the short vertical segments unique to each $S$. Similarly, other pairs of micropatterns said to be discriminable because of terminator differences (e.g., Julesz, 1982, Figure 2; Julesz \& Bergen, 1983, Figure 5) may be discriminable because of the presence of features unique to one of the micropat- 
terns in each pair. In the examples just mentioned, one micropattern in each pair contains a feature that may approximate a crossing texton.

It appears that most, if not all, of the published examples used to support the density-processing model contain additional, confounding features particular to each type of micropattern (e.g., see Bergen \& Julesz, 1983; Julesz, 1981, 1982, 1984a, 1984b; Julesz \& Bergen, 1983). Hence, an alternative to the density-processing model-dubbed the simple detector model-may be proposed. The simple detector model postulates that, rather than counting textons and computing density differences, preattentive processes simply detect the presence of particular features. According to this model, preattentive texture discrimination or target detection arises only when a texture or micropattern contains one or more unique preattentive features relative to a comparison texture or micropattern. Importantly, when micropatterns differ only in the density of textons common to both textures or micropatterns, the simple detector model predicts that preattentive discrimination will not occur. This model represents a limiting case of the density-processing model. That is, the simple detector model holds that (1) if a single texton counting pool is present, preattentive mechanisms simply signal the presence (and possibly sometimes the absence) of a given type of texton, and (2) if multiple pools are present, then density differences are preattentively detected only where the differences are such that one pool detects a type of texton that is not detected by other pools. The aim of the present study was to determine, in the case of terminators, whether the simple detector or the density-processing model best represents preattentive perception.

The following ratio was used as an index of density similarity: density ratio (DR) $=$ (number of terminators in low-density micropattern)/(number of terminators in high-density micropattern). In Julesz's work, micropattern pairs said to be discriminable in terms of terminator density differences have had DRs as high as .67 (i.e., $S$ and E micropatterns; Julesz, 1982, Figure 9), with the lowest nonzero DR being .40 (e.g., Julesz, 1982, Figure 2; Julesz \& Bergen, 1983, Figure 5). In the present study, we therefore used stimuli with DRs in the vicinity of those used by Julesz.

The present study used a task and criteria derived from Treisman's search asymmetry task. This task is argued to be a "feature diagnostic" of preattentive perception (Treisman \& Gelade, 1980; Treisman \& Souther, 1985). With that task it has been found that when preattentive features are present in the target and absent in surrounding distractor micropatterns, then the time taken to determine the presence or absence of a target in an array (RT) increases only slightly with increases in the number of micropatterns (target plus distractors) in the array (Treisman \& Souther, 1985). In preattentive target detection, the distractors have little effect on target detection RT since the target's preattentive feature(s) conspicuously stand out and thus draw focal attention (Julesz,
1981; Treisman \& Souther, 1985). In comparison, when the target is distinguished by the absence of particular preattentive features (e.g., terminators) that are present in the distractors, then RTs tend to be greater and target detection occurs via attentive perception (i.e., sequential shifts of focal attention; Treisman \& Souther, 1985).

In short, attentive target detection is indicated by a large increase in RT as a linear function of the number of micropatterns (target plus distractors) forming the array. We refer to the slope of this increase in RT with array size as the $R T$ gradient. Although preattentive target detection is indicated by a "flat" function, such target detection can also be indicated by small RT gradients (e.g., $10 \mathrm{msec} /$ micropattern; Treisman \& Souther, 1985). Preattentive target detection can result in small nonzero gradients because of "noise" arising as a function of the number of items in the display (Treisman \& Souther, 1985). This effect is comparable to a distraction effect obtained in studies that examined the effects of distractors on letter detection (e.g., Coles, Gratton, Bashore, C. W. Eriksen, \& Donchin, 1985; B. A. Eriksen \& C. W. Eriksen, 1974; C. W. Eriksen \& B. A. Eriksen, 1979; C. W. Eriksen \& Hoffman, 1973; C. W. Eriksen \& St. James, 1986; Grice, Canham, \& Schafer, 1982). The findings of Treisman and coworkers suggest that the upper limit of the flat function corresponds to an RT gradient of about $10 \mathrm{msec} /$ micropattern (Treisman \& Gelade, 1980; Treisman \& Souther, 1985; Treisman, Sykes, \& Gelade, 1977). Hence, that value was used in the present study as a criterion of the upper limit of preattentive target detection.

Using the micropattern pairs shown in Figure 1a, Experiment 1 sought to determine the relationship between DR and the process of target detection (attentive vs. preattentive). In comparison with the distractors, the targets of each pair are distinguished by the presence of a larger number of terminators and the corresponding absence of one line segment. The simple detector model predicts that target detection would be attentive when DR $>0$, whereas the density-processing model predicts that target detectability would be preattentive for some range of nonzero DRs.

If it is assumed that there are a number of different sampling areas for each type of texton, then, within limits, the predictions made here should be independent of the visual angle of the micropatterns used here; that is, it is assumed that there should be a texton (e.g., terminator) counting pool with a sampling area that approximates the size of the micropatterns used. If the density-processing model holds, then such pools would provide maximal sensitivity to density differences; pools significantly smaller or larger than one micropattern would decrease the effective density difference between target and distractors since the counting pools would not be able to identify individual micropatterns.

In Experiment 1, the predictions of the simple detector and the density-processing models were examined at two different viewing distances (and hence different micropattern sizes). The density-processing model 
predicts that if there are multiple sampling areas, then viewing distance should have no significant effect on target detection RT. Similarly, the simple detector model predicts that there should be no effect of viewing distance on RT.

\section{EXPERIMENT 1}

\section{Method}

Subjects. Three experienced psychophysical observers with corrected-to-normal vision participated in the study. Two were the authors, and one was a student volunteer recruited from the University of Melbourne. The latter subject was unaware of the hypotheses being investigated.

Stimuli and Apparatus. As Figure 1a shows, three different pairs of micropatterns (target-distractor pairs) were used. Pairs were labeled according to the number of terminators each possessed. For each pair, target-present and target-absent versions were generated, and three array sizes (target plus distractor) were used (consisting of 4,8 , or 16 micropatterns). The seven terminator (7) micropattern always served as target and, like all micropatterns, was positioned at random within the display matrix. Figure $1 b$ shows an example of a 7-0 target-present display for each of the three array sizes.

Displays were generated by means of a PDP $11 / 73$ computer that controlled Imaging Technology ITEX/Q image processing boards, which, in turn, controlled a C233 Barco color monitor. Micropatterns were white in a black surround, and were oriented at $0^{\circ}$, $90^{\circ}, 180^{\circ}$, or $270^{\circ}$ relative to an arbitrarily selected zero. Mirror reflections were also randomly applied to half of the micropatterns. The space-averaged luminance (measured within the smallest circular region that encompassed an entire micropattern) was measured by a J-16 Tektronix Spot Photometer with a $1^{\circ}$ probe (model $\mathrm{J} 6523-2$ ). The luminance of the line segments and their surround was 32.6 and $3.5 \mathrm{~cd} / \mathrm{m}^{2}$, respectively. The space-averaged luminance of the $0,2,4$, and 7 micropatterns was 8.1, 8.0, 7.9, and $7.9 \mathrm{~cd} / \mathrm{m}^{2}$, respectively. Although the three distractor types $(0,2$, and 4) differed in the length of one of the line segments (see (a)

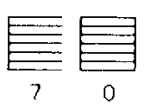

$(D R=.00)$

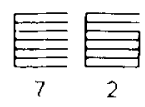

$(D R=.29)$

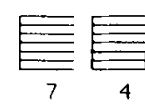

$(D R=.57)$ (b)
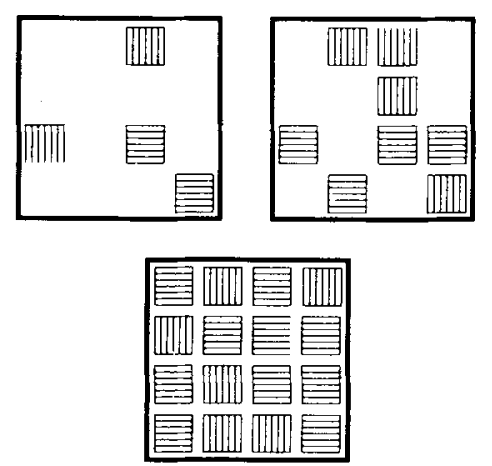

Figure 1. Experiment 1: (a) Target-distractor pairs (7-0, 7-2, and 7-4) and corresponding density ratios (DRs). Micropatterns are labeled according to the number of terminators contained by the target $(7)$ and distractors $(0,2$, or 4). (b) Examples of each of the three array sizes $(4,8$, and 16 micropatterns).
Figure 1a), there were only minor luminance differences across these micropatterns. Line contrast (.81) was constant across all of the micropatterns for all of the experiments in this study.

Procedure. Subjects E.R.H. and S.T. viewed each of the 7-0, $7-2$, and 7-4 stimuli at two viewing distances (825 and 3,575 $\mathrm{mm}$ ), whereas Subject D.R.B. viewed all stimuli at the shorter distance. At both distances, individual terminators and line segments were easily resolved. The dimensions of the stimuli are shown in Table 1 , which shows that the displays were foveal at the longer viewing distance, and were both foveal and parafoveal at the shorter distance (Brown, 1966; Julesz, 1984a). At the nearer distance, the size of each micropattern $\left(2^{\circ} 2^{\prime}\right)$ was such that it was difficult to give focal attention to a single micropattern without scanning eye movements or shifts in focal attention (Julesz, 1984a, 1984b). Micropatterns at both viewing distances were comparable in size to those used in previous studies (cf. Bergen \& Julesz, 1983; Julesz, 1982).

For each target-distractor pair, the various array sizes and target present/absent conditions were interleaved in a quasirandom manner to form blocks of 192 trials. The first 24 trials of each block served as practice trials and were not included in the analyses. The target was present in half of the trials, and each of the three array sizes was represented in an equal proportion (one-third) of the trials.

The subjects were tested individually. Throughout the experiment, the subject's head was steadied by a chinrest. Displays were presented at a rate of one every $7 \mathrm{sec}$, with a warning tone preceding each onset. Each array was displayed until the subject responded by pressing one of two buttons, depending on whether the target was present or absent. The subjects were required to respond as quickly as possible while minimizing errors. Trials eliciting incorrect responses were rerun at the completion of each block. A fixation cross was present between presentations. Each block of trials required approximately $25 \mathrm{~min}$ to complete, and a 10-min break was given between blocks.

For each subject, the order of presentation of the 7-0, 7-2, and $7-4$ blocks was counterbalanced, with viewing distance alternating over successive blocks for Subjects S.T. and E.R.H. At each viewing distance, Subject S.T. received seven repetitions (i.e., seven blocks) of each of the three target-distractor pairs $(8,064$ trials overall). Subject E.R.H. received three repetitions of each pair at each viewing distance $(3,456$ trials), and Subject D.R.B. received three repetitions at the shorter viewing distance only $(1,728$ trials).

\section{Results and Discussion}

The mean proportion of incorrect responses (collapsed across subjects and stimulus conditions) was $4.2 \%$ for target-present stimuli and $0.5 \%$ for target-absent stimuli. In all stimulus conditions, it was found that target-absent RT gradients were generally much larger than the corresponding target-present gradients, suggesting that the detection of target absence required attentive processing. Hence, only the target-present gradients were used to determine which differences In feature density were preattentively detected. Subsequent discussion in this article is therefore confined to the target-present data. Targetabsent data for this experiment, and for Experiments 2 and 3, are presented in the Appendix.

Figure 2 shows target-present RT as a function of array size for the last block of trials of each target-distractor pair. For each subject, these RT distributions were similar to those obtained in prior blocks of trials (see below). The symbols in Figure 2 represent medians, and bar length indicates the interquartile range (25th to 75 th 
Table 1

Experiment 1: Stimulus Dimensions

\begin{tabular}{|c|c|c|c|c|c|c|c|c|}
\hline \multirow{2}{*}{$\begin{array}{l}\text { Viewing } \\
\text { Distance } \\
\text { (in } \mathbf{~ m m} \text { ) }\end{array}$} & \multirow{2}{*}{$\begin{array}{c}\text { Maximum } \\
\text { Array } \\
\text { Size }^{*}\end{array}$} & \multirow{2}{*}{$\begin{array}{c}\text { Micropattern } \\
\text { Size } \\
\end{array}$} & \multirow{2}{*}{$\begin{array}{c}\text { Micropattern } \\
\text { Separation }\end{array}$} & \multirow{2}{*}{$\begin{array}{l}\text { Terminator } \\
\text { Separation } \dagger\end{array}$} & \multirow{2}{*}{$\begin{array}{c}\text { Line } \\
\text { Thickness }\end{array}$} & \multicolumn{3}{|c|}{ Line Segment Lengths } \\
\hline & & & & & & 0 & 2 & 4 \\
\hline $\begin{array}{r}825 \\
3,575 \\
\end{array}$ & $\begin{array}{l}12^{\circ} \\
2^{\circ} 50^{\prime}\end{array}$ & $\begin{array}{r}2^{\circ} 29^{\prime} \\
35^{\prime}\end{array}$ & $\begin{array}{r}37^{\prime} \\
8 \\
\end{array}$ & $\begin{array}{r}17^{\prime} \\
4^{\prime} \\
\end{array}$ & $\begin{array}{l}6^{\prime} \\
1^{\prime}\end{array}$ & $\begin{array}{r}2^{\circ} 29^{\prime} \\
35^{\prime} \\
\end{array}$ & $\begin{array}{r}1^{\circ} 39^{\prime} \\
23^{\prime} \\
\end{array}$ & $\begin{array}{l}50^{\prime} \\
12^{\prime} \\
\end{array}$ \\
\hline
\end{tabular}

*Both arrays and micropatterns were square; thus, the length given is that of each axis of the 16-micropattern display. tEquivalent to the distance between parallel nearest-neighbor line segments within each micropattern.

percentile) of RTs. In the figure, the medians were staggered so as to show the interquartile ranges more clearly (i.e., only the three array sizes shown on the abscissa [4, 8 , and 16 micropatterns] were used). For each block of trials, the medians were calculated from the first 28 trials per array size after the practice trials starting each block. Medians were used instead of means since RT response distributions tended to be skewed, with variances increasing with the magnitude of the means (heteroscedasticity). ${ }^{1}$

The lines shown in the figure represent least-squares regressions, fitted to medians. From these regressions, RT gradients were derived. ${ }^{2}$ The mean (and standard deviation) of the regression $R^{2} \mathrm{~s}$, averaged over subjects and all blocks of the 7-0, 7-2, and 7-4 target-present conditions, was $.95(.10)$. Thus, the linear regressions tended to provide a very good description of the variation of median RT as a function of array size (also see Appendix).

Figure 3 shows RT gradient as a function of the number of blocks of trials presented to each subject. For each target-distractor pair, block numbers correspond to the temporal order or sequence of presentation of blocks. The dashed line shows the 10-msec/micropattern gradient used to indicate the approximate upper limit of the flat func- tion criterion. For S.T. and E.R.H., the even-numbered blocks correspond to stimuli viewed at a distance of $825 \mathrm{~mm}$, and the odd-numbered blocks correspond to a viewing distance of $3,575 \mathrm{~mm}$. (D.R.B. viewed all blocks at a distance of $825 \mathrm{~mm}$.)

Although Figure 3 shows some fluctuation in gradients, only the 7-0 targets for S.T. and D.R.B. were found to consistently approach the flat function criterion. These were the only gradients with a magnitude comparable to the preattentive gradients obtained by Treisman and coworkers (Treisman \& Gelade, 1980; Treisman \& Souther, 1985; Treisman et al., 1977). This suggests that only the 7-0 targets for S.T. and D.R.B. were detected in a preattentive manner. This will be considered in more detail after the effects of practice and viewing distance have been examined.

Figure 3 suggests that practice effects tended to be small. For each subject and target-distractor pair, the change in RT gradient across blocks of trials was computed by using each point in Figure 3 to calculate leastsquares regressions. From these regressions, the rate of change of RT gradient was calculated and used as an index of practice. This rate of change variable was actually

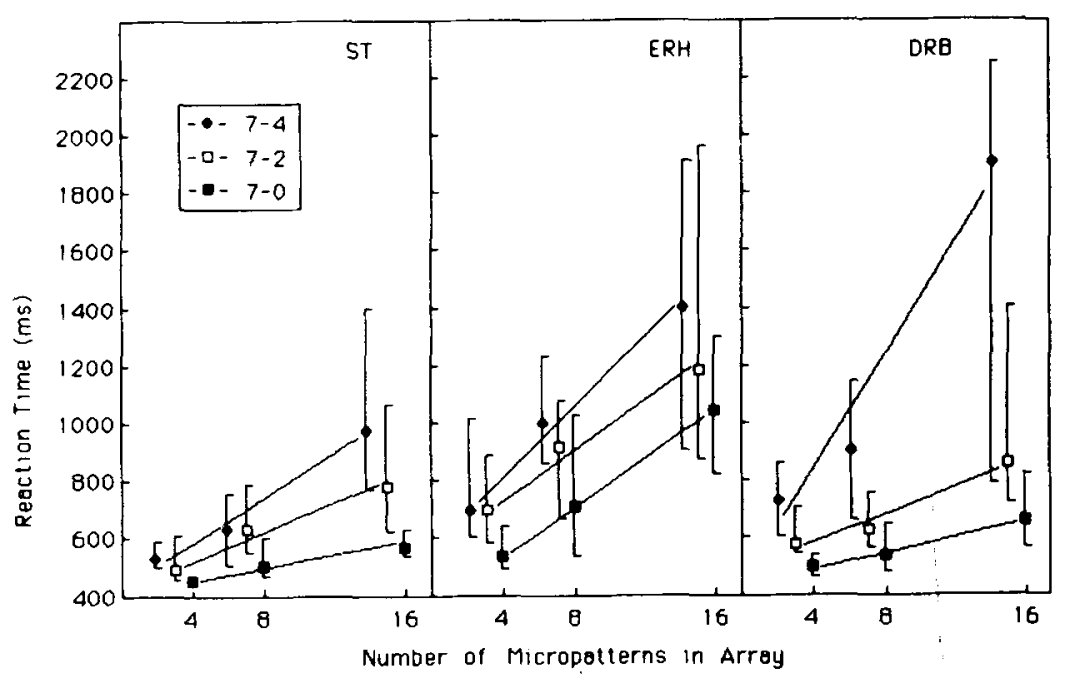

Figure 2. Experiment 1: Target-present reaction time (RT) as a function of array size for the last block of trials of each target-distractor pair. Points represent median RTs, and bars represent interquartile ranges (25th to 75th percentile). Medians each correspond to one of the three array sizes shown, and are staggered only to show the interquartile ranges more clearly. RT gradients were derived from the least-squares regression lines shown. 


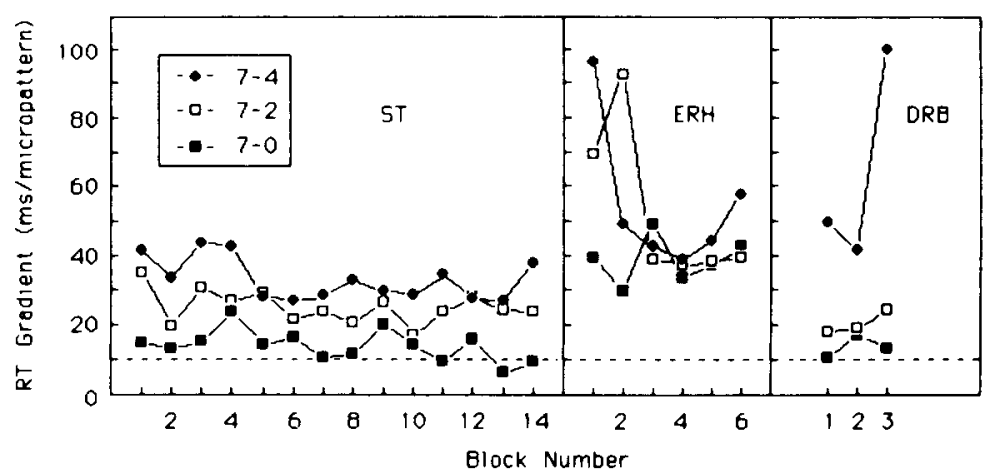

Figure 3. Experiment 1: Reaction time (RT) gradient as a function of the number of blocks of trials presented to each subject. The dashed line corresponds to the 10-msec/micropattern gradients, which was used to indicate the approximate threshold for the flat function criterion.

the gradient of the RT gradients, and so will be referred to as a metagradient. Over all subjects and conditions, the mean metagradient was only $1.8 \mathrm{msec} /$ micropattern per block of trials. Means (and standard deviations) of metagradients, collapsed across subjects, were as follows: $0.5(1.0)$ for $7-0,-2.1(6.2)$ for $7-2$, and $-4.1(3.5)$ for 7-4. In the event that these practice effects are better described by a power function, the log of each gradient was calculated, and the gradient of these log-gradients was derived for each subject and condition. As with the metagradients derived from the untransformed gradients, the metagradients obtained from log-gradients were all close to zero $(M=0.03, S D=0.15)$. For the purposes of this experiment, practice appeared to have little effect on results.

In order to determine the effects of viewing distance, we compared RT gradients in a pairwise manner across viewing distances for each of the 7-0,7-2, and 7-4 stimuli. As mentioned above, Subjects S.T. and E.R.H. received blocks at alternating viewing distances. Since practice effects were found to be small, order (and hence practice) effects were unlikely to be confounded with the effects of viewing distance. To further reduce any influence of practice, only the final blocks were used in the analyses of viewing distance.

For each target-distractor pair, the effects of viewing distance were calculated as follows. Since viewing distance was alternated for a given target-distractor pair, RT gradients were grouped into consecutive pairs, such that each pair contained a gradient from each viewing distance. For each viewing distance and target-distractor pair, the last four blocks of trials for S.T. and the last two blocks of each pair for E.R.H. were used in these analyses. The data from these subjects were then combined to yield six (i.e., four plus two) gradient pairs for each target-distractor pair. The difference between gradients was then obtained for each pair of gradients. For each target-distractor pair, the significance of these differences was determined by means of a $t$ test for matched samples. Results failed to indicate any significant effect of viewing distance
$[7-0, t(5)=0.04 ; 7-2, t(5)=0.10 ; 7-4, t(5)=0.22$ all $p s>.20$ (two-tail)].

Pairwise comparisons between viewing distances also were calculated separately for each subject. For every subject this was done as follows: For each of the 7-0, 7-2, and 7-4 stimuli, the RT gradients from each block were averaged. This resulted in three mean RT gradients (i.e., for 7-0, 7-2, and 7-4) for each viewing distance. For each of the three target-distractor pairs, viewing distance differences (near minus far) were then computed. As with the above-mentioned comparisons, results failed to reveal any significant effects of viewing distance [S.T., $t(2)=$ 2.83; E.R.H., $t(2)=0.29$; all $p s>.10$ (two-tail)]. Thus, results indicated that viewing distance did not significantly influence the RT gradients.

To determine which of the 7-0, 7-2, and 7-4 targets were processed preattentively, analyses were performed for each subject and target-distractor pair to determine the significance of the difference of the gradients from the $10-\mathrm{msec} / \mathrm{micropattern} \mathrm{criterion.} \mathrm{This} \mathrm{was} \mathrm{done} \mathrm{by} \mathrm{cal-}$ culating a least-squares regression using all of the medians obtained for a given target-distractor pair, and comparing the resulting gradient with the $10-\mathrm{msec} /$ micropattern gradient by means of a $t$ test; that is, since practice and viewing distance did not produce significant effects, a single gradient was calculated for each subject and target-distractor pair using all of the medians obtained in that condition. For each subject, a $t$ test was then performed for each of the 7-0, 7-2, and 7-4 conditions. All of the resulting $t$ values were significant, indicating that all gradients were significantly larger than the gradient used as a criterion of the upper limit of the flat function. [For S.T., $t(40)=2.72, p<.01$; for D.R.B., $t(7)=$ $2.64, p=.04$ (two-tail). Gradient $=14 \mathrm{msec} / \mathrm{micro}$ pattern in both cases.] Although these gradients were significantly larger than $10 \mathrm{msec} /$ micropattern, they are still quite small, and may indicate weak preattentive processing. The 7-0 condition for E.R.H. resulted in $t(16)=$ $4.77, p<.05$ (gradient $=38 \mathrm{msec} /$ micropattern). The respective $7-2$ and $7-4$ values were as follows: for S.T., 
$t(40)=4.55$ and $t(40)=7.84$; for E.R.H., $t(16)=4.95$ and $t(16)=2.21 ;$ and for D.R.B., $(t)(7)=6.03$ and $t(7)$ $=3.90$ (all $p \mathrm{~s}<.05$, two-tail).

In summary, the results of Experiment 1 indicated that there was no significant change in RT gradient when micropattern size was changed by a factor of 4.26 (i.e., from $35^{\prime}$ to $2^{\circ} 29^{\prime}$ ) as a result of a change in viewing distance. It was further found that (1) the 7-0 targets may have been only weakly preattentive for S.T. and D.R.B., and not preattentive for E.R.H., and (2) preattentive target detection did not occur when DR $>0$. Overall, it was found that even after considerable practice, preattentive target detection did not occur when DR $>0$. The findings of this experiment fail to offer any clear support to the notion of density processing proposed by Julesz (e.g., 1984a, 1984b).

\section{EXPERIMENT 2}

Despite the evidence suggesting that terminators are preattentive features (e.g., Julesz, 1982; Treisman \& Souther, 1985), preattentive target detection was not observed in the 7-0 condition for E.R.H., and the RT gradients for S.T. and D.R.B. were frequently greater than $10 \mathrm{msec} /$ micropattern (see Figure 3). Detection of the 7-0 targets may not have been strongly preattentive because of particular structural characteristics of the micropatterns that reduced terminator conspicuity (Enns, 1986; Taylor \& Stanley, 1986), thus making terminator detection difficult for observers. Therefore, Experiment 2 was conducted to determine the extent to which the results of Experiment 1 generalized to structurally different stimuli.

The target-distractor pairs used in Experiment 2 are shown in Figure 4a, and examples of the arrays are shown in Figure $4 \mathrm{~b}$. These stimuli were constructed so that the cues to the individual micropatterns were degraded, as demonstrated in Figure $4 \mathrm{~b}$, where it can be seen that the identity of individual micropatterns is difficult to discern when arrays consist of a number of adjacent micropatterns. This is because each of these micropatterns is defined by the spatial relations between the four square components. The separation of components within micropatterns is equal to the separation of components belonging to different, adjacent micropatterns. This arrangement impairs the ease by which individual micropatterns (and hence targets) may be attentively detected, but does not influence preattentive detection (Julesz, 1981, $1982,1984 a, 1984 b)$. This is because attentive perception is highly dependent on the spatial or structural relationships between stimuli, whereas preattentive perception occurs in a manner that has been found to be relatively independent of such relationships (Julesz, 1984b). Degradation was therefore expected to enhance the difference between attentive and preattentive RT gradients.

\section{Method}

The subjects, apparatus, and procedure were the same as in Experiment 1. All stimuli were presented at a distance of $825 \mathrm{~mm}$. Array and micropattern dimensions are shown in Figure 4. Micropatterns were separated by a distance of $21^{\prime}$ of arc (visual angle). Within each micropattern, the nearest neighbor distance between line segments (and hence terminators) was $27^{\prime}$. Line segments were $1^{\circ} 7^{\prime}$ long and $6^{\prime}$ wide. The luminance of the line segments and their surround was 32.6 and $3.5 \mathrm{~cd} / \mathrm{m}^{2}$, respectively. The space-averaged luminances of the $0,3,6$, and 12 micropatterns were $7.7,7.4,7.2$, and $6.5 \mathrm{~cd} / \mathrm{m}^{2}$, respectively.

The task of each subject was to determine whether a given display contained a target of high terminator density (the 12 terminator [12] micropattern in Figure 4a). As Figure 4 shows, the highdensity region of the target micropattern was spread over its four components. To maintain that high-density region, the orientations of the components were held constant within each micropattern. However, micropatterns were rotated, reflected, and positioned according to the same procedure as in Experiment 1. All subjects received three blocks of trials for each of the three target-distractor pairs.

\section{Results and Discussion}

Across subjects and conditions, the mean proportion of incorrect responses was $5.3 \%$ for target-present stimuli and $1.1 \%$ for target-absent stimuli. Figure 5 shows targetpresent RT as a function of array size for the last block of trials of each target-distractor pair for each subject. The RT distributions of the last block were similar to those obtained in earlier blocks, although they tended to result in smaller RTs for the 12-3 and 12-6 conditions (see below). The symbols shown in Figure 5 represent RT medians, and bar length indicates the interquartile range of

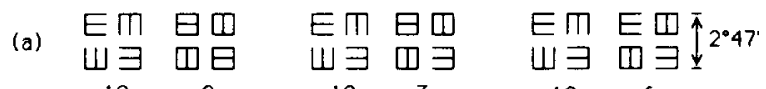

$$
\begin{aligned}
& \begin{array}{llllll}
12 & 0 & 12 & 3 & 12 & 6
\end{array} \\
& (\mathrm{OR}=.00) \quad(\mathrm{DR}=.25) \quad(\mathrm{DR}=.50)
\end{aligned}
$$

(b)
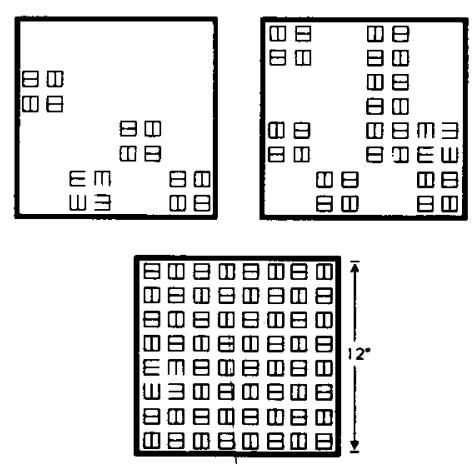

Figure 4. Experiment 2: (a) Target-distractor pairs (12-0, 12-3, and 12-0), labeled according to the number of terminators contained by the target (12) and distractors $(0,3$, or 6). (b) Examples of arrays. At a viewing distance of $825 \mathrm{~mm}$, stimulus dimensions were as shown. 


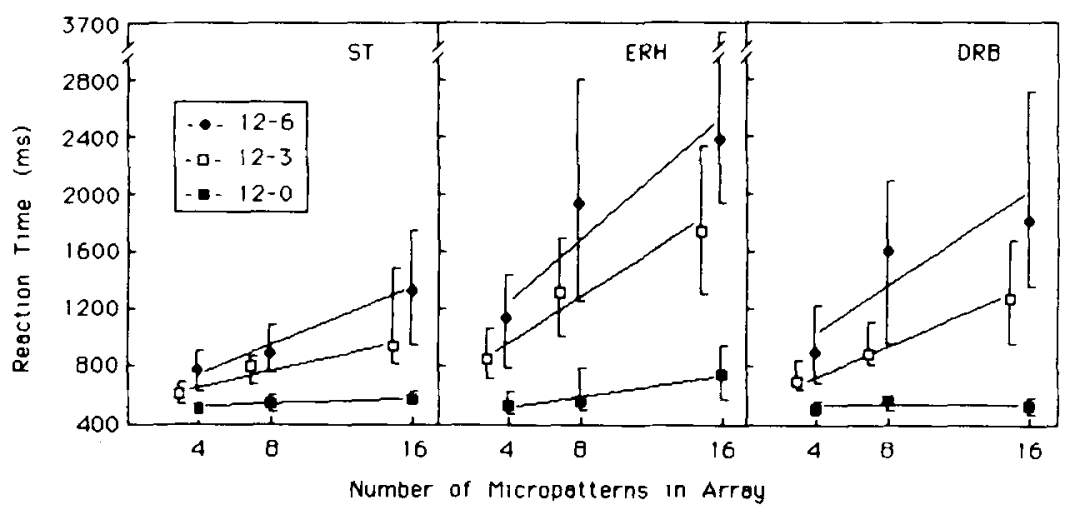

Figure 5. Experiment 2: Target-present reaction time (RT) as a function of array size for the last block of trials of each target-distractor pair. Points represent median RTs, and bars represent interquartile ranges. Medians each correspond to one of the three array sizes shown, but are staggered to show the interquartile ranges more clearly. RT gradients were derived from the least-squares regression lines shown.

RTs. In the figure, the medians are staggered to more clearly show the pattern of interquartile ranges (i.e., each median corresponds to only one of three array sizes; 4 , 8 , or 16 micropatterns). For each block of trials, the medians were calculated from the first 28 trials per array size following the practice trials starting each block.

The lines drawn through the data points in Figure 5 represent least-squares regressions fitted to medians. RT gradients were derived from these regressions. The mean (and standard deviation) of the $R^{2}$ values of the regressions, averaged over subjects and all blocks of the 12-0, $12-3$, and 12-6 target-present conditions, was .87 (.23). $R^{2}$ values were reduced somewhat because there were some nonlinearities observed in the 12-0 condition. The mean (and standard deviation) $R^{2}$ value taken only over the 12-3 and 12-6 conditions was .92 (.16).

Figure 6 shows RT gradient as a function of the number of blocks of trials presented to each subject. Block

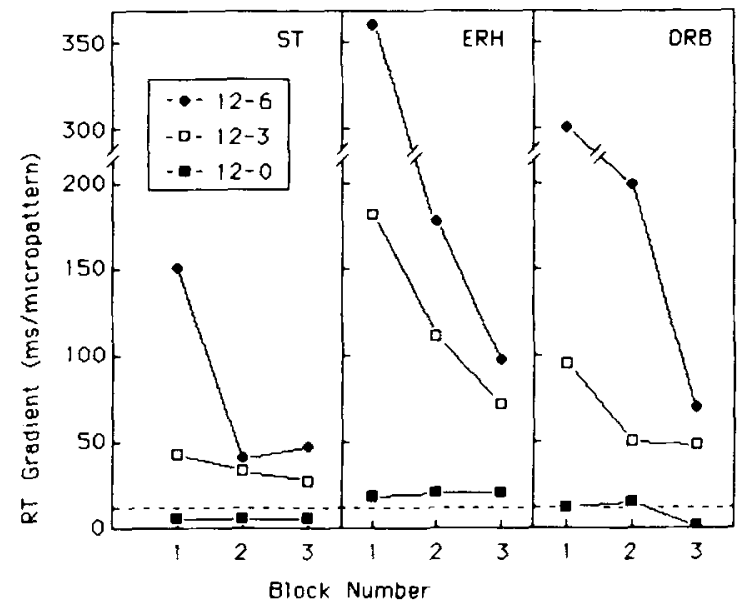

Figure 6. Experiment 2: Reaction time (RT) gradient as a function of the number of blocks of trials presented to each subject. The dashed line indicates the 10 -msec/micropattern criterion for a flat function. numbers correspond to the temporal order or sequence of presentation of the blocks. The dashed line shows the 10-msec/micropattern gradient used to indicate the upper limit for the flat function criterion. Figure 6 shows that only the RT gradients for the $12-0(D R=0)$ stimuli for S.T. and D.R.B. lie in the region considered indicative of preattentive target detection (Treisman \& Gelade, 1980; Treisman \& Souther, 1985; Treisman et al., 1977). The 12-0 gradients for E.R.H. lie above the $10-\mathrm{msec} / \mathrm{micro}$ pattern gradient. However, E.R.H.'s pattern of RT gradients is quite similar to those of S.T. and D.R.B., with the 12-0 gradients being considerably smaller than both the 12-3 and 12-6 gradients.

As in Experiment 1, the significance of the difference of the gradients from the 10-msec/micropattern criterion was examined for each subject and target-distractor pair by means of $t$ tests. In this manner, a single gradient was calculated for each subject and target-distractor pair using all of the medians collected in that condition. (See Experiment 1 for details of the procedure of the analysis.) For D.R.B. and E.R.H., results indicated that the 12-0 gradients were not significantly different from the 10 $\mathrm{msec} / \mathrm{micropattern}$ criterion [for D.R.B., gradient = $9 \mathrm{msec} /$ micropattern, $t(7)=-0.23, p>.05 ;$ for E.R.H., gradient $=20 \mathrm{msec} / \mathrm{micropattern,} t(7)=2.01$, $p>$.05]. Results for S.T. indicated that the 12-0 gradient was significantly smaller than the criterion [obtained gradient $=5 \mathrm{msec} /$ micropattern, $t(7)=-3.29, p<.02$; all $p$ values were two-tail]. For the 12-3 and 12-6 analyses, all gradients were significantly larger than the criterion gradient, with the exception of the 12-3 gradient for D.R.B. $[t(7)=1.82, p=.10]$. The latter result appears to have arisen because of the large size of the estimated standard error of that gradient. Figure 6 shows that 12-3 gradients for D.R.B. averaged $54 \mathrm{msec} / \mathrm{micropat}$ tern, and were all larger than those of S.T. Apart from this questionable exception, the results of the $t$ tests indicate that preattentive target detection occurred only when $\mathrm{DR}=\mathbf{0}$. 
The results of Experiment 2 demonstrate that (1) preattentive target detection was obtained only when $\mathrm{DR}=0$, supporting the simple detector model; (2) preattentive target detection for the $\mathrm{DR}=0$ stimuli occurred with little or no practice (see 12-0 gradients of Block 1 in Figure 6), whereas the attentive targets (12-3 and 12-6) failed to become preattentive even after considerable practice; and (3) the failure to support the density-processing model found in Experiment 1 generalized to the structurally different stimuli used in Experiment 2 . The most salient difference in the results of the two experiments concerns the difference between the RT gradients for the DR $=0$ targets. For all subjects, the 120 gradients were considerably smaller than the 7-0 gradients (e.g., 20 and $42 \mathrm{msec} / \mathrm{micropattern,} \mathrm{respectively,}$ for E.R.H.), despite the fact that practice effects were found to be weak. This suggests that terminator conspicuity may have been affected by structural parameters of the micropatterns, such as the presence of local highdensity differences in blob orientation produced by the line segments within each of the Figure la micropatterns.

\section{EXPERIMENT 3}

Failure to support the density-processing model may have been a function of the absolute number of textons present in each of the micropatterns used in Experiments 1 and 2; that is, if preattentive density-processing mechanisms have a low capacity for counting the number of textons within each micropattern, then terminator density differences across micropatterns would not be detected. This would be because the number of textons in the lower density distractors (e.g., 2 for 7-2, 4 for 7-4) matched or exceeded the number of terminators that could be counted within each micropattern. Hence, Experiment 3 was conducted using DRs of .25 and .50 , where the targets contained four terminators and the distractors contained only one or two terminators.

\section{Method}

The subjects consisted of those used previously, plus 3 additional subjects. The latter 3 were students from the University of Melbourne who were included in order to determine the extent to which results of the present experiment generalized to subjects not having extensive practice with the target detection task. All of the additional subjects had normal vision and were unaware of the hypotheses being investigated.

The target-distractor pairs, labeled 4-1 and 4-2, are shown in Figure 7a. As before, three array sizes were used (4, 8, and 16 micropatterns). Examples of the 16-micropattern target-present arrays are shown in Figure $7 \mathrm{~b}$. The apparatus and procedure of stimulus presentation were the same as those used in Experiments 1 and 2. All stimuli were viewed at a distance of $825 \mathrm{~mm}$, with stimulus dimensions shown in Figure 7. Of these micropatterns, the inner segments were $50^{\prime}$ long, and all line segments were $6^{\prime}$ in thickness. The luminances of the line segments and surround were 32.6 and $3.5 \mathrm{~cd} / \mathrm{m}^{2}$, respectively. The space-averaged luminances of the 1,2 , and 4 micropatterns were $4.8,4.9$, and $4.9 \mathrm{~cd} / \mathrm{m}^{2}$, respectively.

Subjects S.T., E.R.H., and D.R.B. each received three blocks of each of the 4-1 and 4-2 target-distractor pairs. Each of the remaining subjects was presented with two blocks of each of these

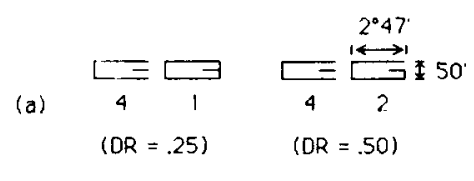

(b)

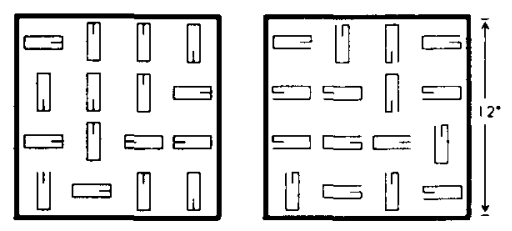

Figure 7. Experiment 3: (a) Target-distractor pairs (4-1 and 4-2), labeled according to the number of terminators possessed by the target (4) and distractors (1 or 2). (b) Examples of arrays. At a viewing distance of $825 \mathrm{~mm}$, stimulus dimensions were as shown.

stimuli. As before, the first 24 trials of each block served as practice trials.

\section{Results and Discussion}

Median RTs were obtained for each array size and target-distractor combination. The mean proportion of incorrect responses (collapsed across all subjects) was $2.0 \%$ for 4-1 and 2.7\% for 4-2. As in Experiments 1 and 2, least-squares regressions were fitted to RT medians to obtain the target-present RT gradients for each block of trials. The mean (and standard deviation) of the regression $R^{2}$ s, averaged over all blocks of the 4-1 and 4-2 target-present conditions, was $.95(.05)$ for the highly practiced subjects and $.95(.06)$ for the relatively unpracticed subjects.

Figure 8 shows RT gradient as a function of the number of blocks of trials presented to each subject. For each target-distractor pair, the block numbers correspond to the temporal order in which blocks were presented. The dashed line shows the 10-msec/micropattern flat function criterion. As in Experiments 1 and 2, the significance of the difference of the gradients from $10 \mathrm{msec} / \mathrm{micropat}-$ tern was examined for each subject and target-distractor pair by means of $t$ tests. For each of the blocks presented to S.T., E.R.H., and D.R.B., a single gradient was calculated for each target-distractor pair using all of the medians collected in each condition. (See Experiment 1 for details of the procedure of analysis.) Results for the practiced subjects indicated that except in the case of the 4-1 conditions for S.T. and D.R.B., all gradients were significantly greater than the criterion (at $p<.05$, two tail). The 4-1 gradients for S.T. and D.R.B. were not statistically different from the 10 -msec/micropattern gradient. For the 4-1 and 4-2 conditions, respectively, $t(7)$ values are as follows: for S.T., 0.89 and 2.71; for E.R.H., 5.15 and 2.81 ; D.R.B., 1.97 and $3.15[t(7)=2.37, p>.05$, two-tail].

The same form of analysis was performed for the unpracticed subjects. Because of the small number of blocks presented to each of these subjects, data were collapsed across subjects; that is, the 4-1 and 4-2 medians were com- 


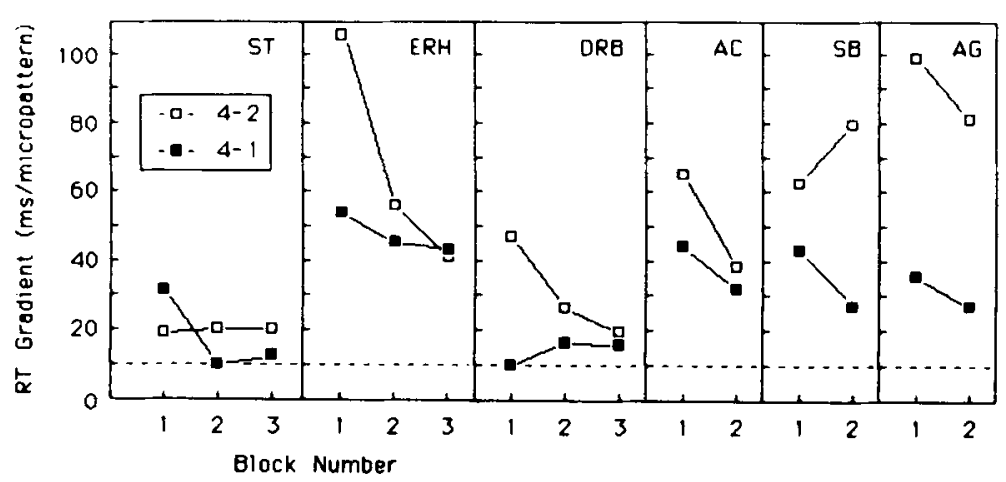

Figure 8. Experiment 3: Reaction time (RT) gradient as a function of the number of blocks of trials presented to each subject. The dashed line indicates the 10msec/micropattern criterion for a flat function.

bined across subjects to yield two $t$ values [for 4-1, $t(16)$ $=5.7, p<.001$, and for $4-2, t(16)=8.24, p<.001$, two-tail]. Averaged across the gradients obtained for the unpracticed subjects, the mean gradients for the 4-1 and 4-2 conditions were 32 and $72 \mathrm{msec} / \mathrm{micropattern}$, respectively. These results indicate that for the unpracticed subjects, the 4-1 and 4-2 RT gradients were significantly greater than $10 \mathrm{msec} / \mathrm{micropattern}$.

Overall, the results of Experiment 3 show that preattentive target detection occurred only in the 4-1 condition for S.T. and D.R.B., with gradients of 12 and $16 \mathrm{msec} / \mathrm{micropattern}$, respectively. For the remaining subjects and conditions, target detection was not preattentive. Although those results again generally support the simple detector model over the density-processing model, it appears that density processing may occur under conditions in which (1) subjects have had extensive practice with the target-detection task, and (2) stimuli have low DRs (i.e., large density differences) and distractors have low terminator numbers.

\section{GENERAL DISCUSSION}

In this study using a number of stimuli, viewing distances, and density differences, results generally failed to support Julesz's (e.g., 1980, 1982, 1984a, 1984b) claims of instantaneous terminator density processing, as described by the density-processing model. Our results suggest than when terminators are preattentively detected, preattentive processes usually signal only the presence of terminators, as predicted by the simple detector model. If density processing does occur, results indicate that it is limited to particular highly practiced subjects and occurs only for micropatterns with large density differences (i.e., DRs $\leq .25$ ) and for distractors with low terminator numbers. ${ }^{3}$

Limited support was found for the density-processing model, however, and is worthy of some comment. The simple detector model is a limiting case of the densityprocessing model (i.e., density differences computed only when $D R=0$ ), and it may be argued that results support a density-processing model that is highly constrained ac- cording to the level of practice observers receive, the number of textons in the micropatterns, and DR. However, it also may be that the limited support for the densityprocessing model was a result of highly practiced subjects being able to learn feature conjunctions under certain conditions (Treisman \& Souther, 1985; Treisman et al., 1977). Although this issue must await further investigation, it is important to note that the current findings offer far more support for the simple detector model than for the density-processing model.

The present findings further suggest that Julesz's examples of density processing [e.g., Julesz, 1982, Figure 2 $(\mathrm{DR}=.67)$ and Figure $9(\mathrm{DR}=.40)$; Julesz \& Bergen, 1983, Figure $5(\mathrm{DR}=40)$ ] have $\mathrm{DRs}$ that are too large for preattentive target detection or texture discrimination to occur for most practiced and unpracticed subjects; that is, the density differences in Julesz's examples are probably too small for density processing to occur. Our results, however, do suggest that density processing may be important for attentive perception. This observation derives from the fact that most of the attentive target-present RT gradients increased with DR (see Figures 3, 6, and 8, and Appendix).

With regard to future research, a number of issues may be raised. First, the sample size in our experiments was small, and individual differences were apparent. This may limit the generality of the conclusions of this study. Although the findings reported here require replication using larger sample sizes, it is notable that the subjects of the present study displayed considerable consistency in that the simple detector model was generally supported. Second, it may be argued that the failure to support the density-processing model may have been a function of micropattern size. In Experiment 1, it was found that there was no significant change in RT gradient when micropattern size was changed by a factor of 4.26 as a result of a change in viewing distance, with results supporting the simple detector model at both distances. It remains to be seen, however, whether the simple detector model is able to account for results using micropattern sizes different from those used here. In the present study, micropattern size was not varied independently of micropattern sepa- 
ration. Thus, it also remains to be determined whether micropattern separation is a critical variable in the processing of texton density.

Although the present results generally failed to support the density-processing model that assumes that the sampling areas of the relevant texton pools cover domains such as that of a single micropattern, the present findings do not rule out other versions of the density-processing model. This is because the sampling area of a texton counting pool may be defined in a number of ways. It may be that some absolute or relative distance between textons is important in defining the area. Textons may be counted as belonging to a given pool only if they satisfy certain constraints, for example, (1) if they are in groups of less than some specific number and are less than a particular distance apart, or (2) if they are clustered into pools according to some specific grouping statistic based on relative distances. Although these variants of the densityprocessing model would have difficulty accounting for the present findings (since targets with DRs $>0$ were generally not preattentively detected), there may be conditions under which a density-processing model is supported. Clearly, these alternatives merit further investigation.

Another issue concerns the question of whether density processing occurs for other classes of textons. For crossing textons, this could be investigated by using a design similar to that used in the experiments reported here. For blobs, the issue is more complex. Although density differences in blob textons have been shown to be associated with texture discriminability (e.g., Pratt, Faugeras, \& Gagalowicz, 1978), it has yet to be established that texture discrimination or target detection occurs on the basis of processes that count instances of blobs. It may be that when blobs are the critical textons, texture discrimination or target detection arises from mechanisms that operate globally over the image and are sensitive only to large luminance (energy) differences. To decide between these alternatives, it is necessary to vary blob density without altering overall luminance. This may be done by constructing micropatterns from contiguous pairs of blobs or bars, with the space-averaged luminance of each micropattern corresponding to that of the surround (e.g., black-and-white contiguous bars embedded in a grey surround). With such micropatterns, the processing of blob density may be investigated using a texture discrimination paradigm. The findings for blobs and crossings may then be integrated with the findings of the present study-that preattentive density processing does not generally occur for terminators-in order to build a comprehensive model of the processing of texton density in preattentive perception.

\section{REFERENCES}

Bergen, J. R., \&ulesz, B. (1983). Rapid discrimination of visual patterns. IEEE Transactions on Systems, Man, \& Cybernetics, 13, 857-863.

Brown, J. L. (1966). The structure of the visual system. In C. H. Graham (Ed.), Vision and visual perception (pp. 39-59). New York: Wiley.
Coles, M. G. H., Gratton, G., Bashore, T. R., Eriksen, C. W., \& Donchin, E. (1985). A psychophysiological investigation of the continuous flow model of human information processing. Journal of Experimental Psychology: Human Perception \& Performance, 11, 143-149.

EDWARDS, A. L. (1955). Statistical methods for the behavioral sciences. New York: Rinehart.

ENNs, J. (1986). Seeing textons in context. Perception \& Psychophysics, 39, 143-147.

ERIKSEN, B. A., \& ERIKSEN, C. W. (1974). Effects of noise letters on the identification of a target letter in a nonsearch task. Perception \& Psychophysics, 16, 143-149.

ERIKSEN, C. W., ERIKSEN, B. A. (1979). Target redundancy in visual search: Do repetitions of the target within the display impair processing? Perception \& Psychophysics, 26, 195-205.

ERIKSEN, C. W., HofFMAN, J. E. (1973). The extent of processing of noise elements during selective coding from visual displays. Perception \& Psychophysics, 14, 155-160.

ErIKSEN, C. W., \& ST. JAmes, J. D. (1986). Visual attention within and around the field of focal attention: A zoom lens model. Perception \& Psychophysics, 40, 225-240.

Grice, G. R., Canham, L., \& Schafer, C. (1982). Development of associative perceptual interference. Perception \& Psychophysics, 32 , 375-387.

Hurlbert, A., \& Poggio, T. (1985). Spotlight on attention. Trends in Neurosciences, 8, 309-311.

JuLESZ, B. (1980). Spatial nonlinearities in the instantaneous perception of textures with identical power spectra. Philosophical Transactions of the Royal Society of London B, 290, 83-94.

Julesz, B. (1981). Textons, the elements of texture perception, and their interactions. Nature, 290, 91-97.

JuLESz, B. (1982). The role of terminators in preattentive perception of line textures. In D. G. Albrecht (Ed.), Lecture notes in biomathematics: 44. Recognition of pattern and form (pp. 33-58). New York: Springer-Verlag.

Julesz, B. (1984a). Adaptation in a peephole: A texton theory of preattentive vision. In L. Spillman \& B. R. Wooten (Eds.), Sensory experience, adaptation, and perception: Festschrift for Ivo Kohler (pp. 37-52). Hillsdale, NJ: Erlbaum.

JuLESZ, B. (1984b). Toward an axiomatic theory of preattentive vision. In G. M. Edelman, W. E. Gall, \& W. M. Cowan (Eds.), Dynamic aspects of neocortical function (pp. 585-612). New York: Wiley.

Julesz, B., \& Bergen, J. R. (1983). Textons, the fundamental elements in preattentive vision and perception of textures. Bell System Technical Journal, 62, 1619-1645.

Pratt, W. K., Faugeras, O. D., Gagalowicz, A. (1978). Visual discrimination of stochastic texture fields. IEEE Transactions on Systems, Man, \& Cybernetics, 8, 796-804.

TAYLOR, S. (1986). Real and virtual features in texture perception. Perceptual \& Motor Skills, 63, 1300-1302.

TAYLOR, S., STANLEY, G. (1986). Contrast and conspicuity in preattentive texture perception. Perception \& Psychophysics, 40, 287289.

Treisman, A., \& Gelade, G. (1980). A feature integration theory of attention. Cognitive Psychology, 12, 97-136.

Treisman, A., \& Souther, J. (1985). Search asymmetry: A diagnostic for preattentive processing of separable features. Journal of $E x$ perimental Psychology: General, 114, 285-310.

Treisman, A., Sykes, M., \& Gelade, G. (1977). Selective attention and stimulus integration. In S. Dornic (Ed.), Attention and performance VI (pp. 333-361). Hillsdale, NJ: Erlbaum.

\section{NOTES}

1. Compared with mean-based gradients, the gradients obtained from medians were more stable since medians are less influenced by outliers. For low RT gradients, however, means and medians produced very similar results. For median-based RT gradients less than or equal to $20 \mathrm{msec} /$ micropattern, for example, the difference between the meanand median-based RT gradient was calculated for each block. The mean of those differences was only $1.1 \mathrm{msec} /$ micropattem $(S D=2.4)$. 
2. The pattern of gradients reported in this and the following experiments remained unchanged when regressions were fitted to the raw data rather than to the medians.

3. The subjects also received blocks of trials of an isotexton targetdistractor pair consisting of a $\mathrm{T}$-shaped target and L-shaped distractors. In each of those micropatterns, the constituent line segments were separated by gaps, giving the target and distractors four terminators each.
These isotexton stimuli were used as a measure of the speed of attentive target detection (see Bergen \& Julesz, 1983). The RTs of the target-distractor pairs used in Experiments 1-3 were compared with the RTs obtained in the T-L condition by means of ANOVAs using raw and transformed data $\left\{\right.$ transformation: $\left.\mathrm{RT}^{\prime}=[\log (\mathrm{RT})]^{-1}\right\}$. Again, the simple detector model was supported over the density-processing model in all conditions except for the 4-1 condition for S.T. and D.R.B.

APPENDIX

Linear Regressions, and the Variance Each Accounts for $\left(R^{2}\right)$, Fitted to the Medians from the Last Block of Trials for Each Subject and Condition

\begin{tabular}{|c|c|c|c|c|c|c|}
\hline \multirow[b]{2}{*}{ Subject } & \multirow[b]{2}{*}{ Condition } & \multirow[b]{2}{*}{ Target } & \multicolumn{2}{|c|}{ Regression Equation } & \multirow[b]{2}{*}{$R^{2}$} & \multirow[b]{2}{*}{$F$} \\
\hline & & & Gradient & Intercept & & \\
\hline \multicolumn{7}{|c|}{ Experiment 1} \\
\hline S.T. & $\begin{array}{l}7-0 \\
7-2 \\
7-4\end{array}$ & $\begin{array}{l}\mathbf{P} \\
\mathbf{A} \\
\mathbf{P} \\
\mathbf{A} \\
\mathbf{P} \\
\mathbf{A}\end{array}$ & $\begin{array}{r}9.18 \\
47.84 \\
23.61 \\
111.46 \\
37.95 \\
-38.50\end{array}$ & $\begin{array}{l}417.00 \\
320.50 \\
410.00 \\
275.00 \\
354.50 \\
175.52\end{array}$ & $\begin{array}{l}.982 \\
.997 \\
.969 \\
.999 \\
.989 \\
.996\end{array}$ & $\begin{array}{l}6.20^{*} \\
2.61 \\
0.08 \\
0.24 \\
1.88 \\
0.66\end{array}$ \\
\hline E.R.H. & $\begin{array}{l}7-0 \\
7-2 \\
7-4\end{array}$ & $\begin{array}{l}\mathbf{P} \\
\mathbf{A} \\
\mathbf{P} \\
\mathbf{A} \\
\mathbf{P} \\
\mathbf{A}\end{array}$ & $\begin{array}{r}42.34 \\
80.50 \\
39.25 \\
132.20 \\
57.75 \\
83.39\end{array}$ & $\begin{array}{r}361.50 \\
555.00 \\
562.00 \\
595.50 \\
490.00 \\
1,117.00\end{array}$ & $\begin{array}{l}.999 \\
.998 \\
.983 \\
.999 \\
.991 \\
.999\end{array}$ & $\begin{array}{l}0.10 \\
0.25 \\
0.08 \\
0.00 \\
0.27 \\
5.34^{*}\end{array}$ \\
\hline D.R.B. & $\begin{array}{l}7-0 \\
7-2 \\
7-4\end{array}$ & $\begin{array}{l}\mathbf{P} \\
\mathbf{A} \\
\mathbf{P} \\
\mathbf{A} \\
\mathbf{P} \\
\mathbf{A}\end{array}$ & $\begin{array}{r}13.39 \\
58.29 \\
24.14 \\
157.29 \\
100.89 \\
132.57\end{array}$ & $\begin{array}{l}447.00 \\
452.00 \\
470.00 \\
441.00 \\
241.00 \\
905.00\end{array}$ & $\begin{array}{l}.992 \\
.973 \\
.971 \\
.977 \\
.961 \\
.898\end{array}$ & $\begin{array}{c}0.74 \\
0.27 \\
0.51 \\
3.98^{*} \\
0.25 \\
17.13^{*}\end{array}$ \\
\hline \multicolumn{7}{|c|}{ Experiment 2} \\
\hline S.T. & $\begin{array}{l}12-0 \\
12-3 \\
12-6\end{array}$ & $\begin{array}{l}\mathbf{P} \\
\mathbf{A} \\
\mathbf{P} \\
\mathbf{A} \\
\mathbf{P} \\
\mathbf{A}\end{array}$ & $\begin{array}{r}5.34 \\
5.93 \\
26.48 \\
101.84 \\
46.91 \\
220.89\end{array}$ & $\begin{array}{l}458.50 \\
532.00 \\
528.50 \\
833.50 \\
549.50 \\
592.00\end{array}$ & $\begin{array}{l}.560 \\
.950 \\
.934 \\
.959 \\
.979 \\
.961\end{array}$ & $\begin{array}{l}0.11 \\
0.16 \\
0.55 \\
4.82^{*} \\
0.35 \\
6.19^{*}\end{array}$ \\
\hline E.R.H. & $\begin{array}{l}12-1 \\
12-3 \\
12-6\end{array}$ & $\begin{array}{l}\mathbf{P} \\
\mathbf{A} \\
\mathbf{P} \\
\mathbf{A} \\
\mathbf{P} \\
\mathbf{A}\end{array}$ & $\begin{array}{r}19.79 \\
19.77 \\
70.89 \\
228.63 \\
97.18 \\
227.96\end{array}$ & $\begin{array}{r}419.00 \\
757.50 \\
632.00 \\
905.50 \\
896.00 \\
1,262.00\end{array}$ & $\begin{array}{l}.959 \\
.961 \\
.953 \\
.938 \\
.883 \\
.966\end{array}$ & $\begin{array}{l}0.88 \\
0.07 \\
2.26 \\
6.78^{*} \\
1.42 \\
5.61^{*}\end{array}$ \\
\hline D.R.B. & $\begin{array}{l}12-0 \\
12-3 \\
12-6\end{array}$ & $\begin{array}{l}\mathbf{P} \\
\mathbf{A} \\
\mathbf{P} \\
\mathbf{A} \\
\mathbf{P} \\
\mathbf{A}\end{array}$ & $\begin{array}{r}0.79 \\
13.29 \\
47.61 \\
311.38 \\
68.57 \\
475.32\end{array}$ & $\begin{array}{r}521.00 \\
640.00 \\
505.00 \\
149.50 \\
792.00 \\
-31.00\end{array}$ & $\begin{array}{l}.020 \\
.690 \\
.999 \\
.997 \\
.772 \\
.998\end{array}$ & $\begin{array}{l}3.92 \\
5.80 * \\
0.01 \\
1.08 \\
0.78 \\
0.89\end{array}$ \\
\hline \multicolumn{7}{|c|}{ Experiment 3} \\
\hline S.T. & 4-1 & $\begin{array}{l}\mathbf{P} \\
\mathbf{A} \\
\mathbf{P} \\
\mathbf{A}\end{array}$ & $\begin{array}{r}12.34 \\
19.63 \\
20.21 \\
122.05\end{array}$ & $\begin{array}{l}395.50 \\
441.50 \\
452.00 \\
387.50\end{array}$ & $\begin{array}{l}.969 \\
.990 \\
.961 \\
.985\end{array}$ & $\begin{array}{l}0.08 \\
1.84 \\
0.04 \\
1.24\end{array}$ \\
\hline
\end{tabular}




\begin{tabular}{|c|c|c|c|c|c|c|}
\hline \multirow[b]{2}{*}{ Subject } & \multirow[b]{2}{*}{ Condition } & \multirow[b]{2}{*}{ Target } & \multicolumn{2}{|c|}{ Regression Equation } & \multirow[b]{2}{*}{$R^{2}$} & \multirow[b]{2}{*}{$F$} \\
\hline & & & Gradient & Intercept & & \\
\hline E.R.H. & $\begin{array}{l}4-1 \\
4-2\end{array}$ & $\begin{array}{l}\mathbf{P} \\
\mathbf{A} \\
\mathbf{P} \\
\mathbf{A}\end{array}$ & $\begin{array}{r}42.82 \\
107.82 \\
40.93 \\
90.38\end{array}$ & $\begin{array}{l}378.00 \\
421.00 \\
512.00 \\
964.50\end{array}$ & $\begin{array}{l}.998 \\
.973 \\
.979 \\
.989\end{array}$ & $\begin{array}{l}1.13 \\
6.42^{*} \\
0.00 \\
0.04\end{array}$ \\
\hline D.R.B. & $\begin{array}{l}4-1 \\
4-2\end{array}$ & $\begin{array}{l}\mathbf{P} \\
\mathbf{A} \\
\mathbf{P} \\
\mathbf{A}\end{array}$ & $\begin{array}{l}15.55 \\
59.59 \\
19.45 \\
78.82\end{array}$ & $\begin{array}{l}443.50 \\
379.50 \\
485.50 \\
666.00\end{array}$ & $\begin{array}{l}.990 \\
.983 \\
.947 \\
.984\end{array}$ & $\begin{array}{l}0.23 \\
2.44 \\
0.15 \\
3.12\end{array}$ \\
\hline A.C. & $\begin{array}{l}4-1 \\
4-2\end{array}$ & $\begin{array}{l}\mathbf{P} \\
\mathbf{A} \\
\mathbf{P} \\
\mathbf{A}\end{array}$ & $\begin{array}{r}32.05 \\
103.52 \\
38.46 \\
116.82\end{array}$ & $\begin{array}{l}438.50 \\
387.50 \\
552.00 \\
615.00\end{array}$ & $\begin{array}{l}.999 \\
.986 \\
.940 \\
.999\end{array}$ & $\begin{array}{l}0.07 \\
3.03 \\
0.27 \\
0.36\end{array}$ \\
\hline S.B. & $\begin{array}{l}4-1 \\
4-2\end{array}$ & $\begin{array}{l}\mathbf{P} \\
\mathbf{A} \\
\mathbf{P} \\
\mathbf{A}\end{array}$ & $\begin{array}{r}27.07 \\
98.95 \\
79.32 \\
131.80\end{array}$ & $\begin{array}{l}740.00 \\
833.50 \\
326.00 \\
596.50\end{array}$ & $\begin{array}{l}.760 \\
.983 \\
.996 \\
.995\end{array}$ & $\begin{array}{l}0.00 \\
0.81 \\
0.50 \\
3.99 *\end{array}$ \\
\hline A.G. & $\begin{array}{l}4-1 \\
4-2\end{array}$ & $\begin{array}{l}\mathbf{P} \\
\mathbf{A} \\
\mathbf{P} \\
\mathbf{A}\end{array}$ & $\begin{array}{r}35.70 \\
87.18 \\
98.59 \\
147.52\end{array}$ & $\begin{array}{l}391.50 \\
260.00 \\
271.50 \\
679.50\end{array}$ & $\begin{array}{l}.981 \\
.997 \\
.968 \\
.999\end{array}$ & $\begin{array}{l}0.00 \\
3.13 \\
0.35 \\
0.92\end{array}$ \\
\hline
\end{tabular}

Note-Both target-present (P) and target-absent (A) regressions are given. Regression equations are of the form $y=m x+c$, where $y=$ reaction time (in msec), $m=\mathbf{R T}$ gradient, $x=$ number of micropatterns in array, and $c=y$ intercept (in $\mathrm{msec}$ ). For each regression, analyses of variance (ANOVAs) were performed, testing for linearity of regression according to a procedure described by Edwards (1955). For each subject and condition, ANOVAs were based on the RTs of all trials of the last block (i.e., 84 trials for each regression). $F(1,81)$ values greater than 3.96 indicate significant departures from linearity at alpha $=.05$. It can be seen that for the target-present conditions (upon which all of the analyses of the present study were based), almost all of the $F$ values were nonsignificant, indicating that a linear function provides a good account of the relationship between RT and array size. This conclusion is further supported by the fact that most of the $R^{2}$ values are quite large. $\quad{ }^{*} p<.05$.

(Manuscript received September 8, 1987; revision accepted for publication July 6,1988 .) 